\title{
AlmaCenamiento de Semillas y Germinación de StenOCEREus THURBERI, UNA CACTÁCEA CON VIVIPARIDAD FACULTATIVA
}

\author{
Santos Bersaín Pérez-González ${ }^{1}$, Álvaro Reyes-Olivas², Edmundo García-Moya ${ }^{1}$, \\ angélica Romero-Manzanares ${ }^{1,3}$, José Rodolfo García-Nava ${ }^{1}$, \\ Gabriel A. Lugo-García Y y Bardo Sánchez-Soto² \\ ${ }^{1}$ Colegio de Postgraduados, Montecillo, Texcoco, Estado de México. México \\ ${ }^{2}$ Universidad Autónoma de Sinaloa, Escuela Superior de Agricultura del Valle del Fuerte, Ahome, Sinaloa, México. \\ ${ }^{3}$ Autor para la correspondencia: dahly@colpos.mx
}

\begin{abstract}
Resumen: La estrategia de germinación es fundamental para asegurar la supervivencia de las poblaciones naturales, mientras que el almacenamiento y la viabilidad de las semillas son importantes en programas de repoblación y cultivo. Ambos aspectos están poco estudiados en cactáceas arborescentes vivíparas del Desierto Sonorense. Esta investigación evaluó la germinación de Stenocereus thurberi en plantas no vivíparas y vivíparas; también se estudió el efecto del almacenamiento en semillas remanentes de frutos que presentaron viviparidad, comparada con el comportamiento de la semilla de progenitores no vivíparos. La respuesta en tiempo de inicio $\left(\mathrm{T}_{\mathrm{i}}\right)$ y tiempo medio de germinación $\left(\mathrm{T}_{50}\right)$, así como el potencial germinativo (PG) de las semillas, fueron analizados bajo un diseño de bloques al azar con cuatro repeticiones, en cajas bomboneras (bloques) con unidades experimentales de 50 semillas. Los resultados confirmaron mayor velocidad de germinación y alta tolerancia al almacenamiento en las semillas de frutos caracterizados por viviparidad, a la vez que mostraron variación y reducción del porcentaje de germinación con respecto a las semillas de plantas no vivíparas. La germinación precoz combinada con la alta tolerancia del embrión a la desecación son condiciones extraordinarias y muy raras en el reino vegetal, que favorecen el establecimiento de las plantas al permitir que las plántulas ganen vigor desde antes de que ocurra la dispersión.
\end{abstract}

Palabras clave: almacenamiento, cripto-viviparidad, germinación precoz, potencial germinativo.

\begin{abstract}
The strategy of germination is central to ensure survivorship of natural populations. Storage and viability of seeds are also important features in regeneration and cropping. Both aspects of viviparous arborescent cacti of Sonoran Desert are lacking. This research focused on the germination of Stenocereus thurberi in viviparous and non-viviparous plants. Also, the effect of storage in the remaining seeds of fruits having viviparous seeds as compared with the behavior of seeds of non-viviparous plants. The response in the initiation $\left(\mathrm{T}_{\mathrm{i}}\right)$, mean initiation time $\left(\mathrm{T}_{50}\right)$, and the germinative potential $(\mathrm{GP})$ of seeds were analyzed with a randomized block design, four replicates in candy boxes with 50 seeds as experimental units. The results confirmed a greater velocity of germination and high tolerance to storage in seeds of fruits having viviparous seeds. Meanwhile they showed variation in the reduction of percentage of germination as compared with seeds from fruits on non-viviparous plants. The precocity of germination combined with the high tolerance to drying are outstanding and rare traits in Plant Kingdom, which favors seedlings establishment due to gain vigor before the initiation of dispersal.
\end{abstract}

Keywords: crypto-viviparity, germinative potential, precocious germination, seed storage.

$\mathbf{E}$ n los hábitats fluviales, inundables o costeros, las plantas halófitas han desarrollado adaptaciones asociadas a la supervivencia y reproducción; las primeras están basadas en la tolerancia y eliminación de sales, y las reproductivas en adaptaciones embriológicas como la germinación vivípara y cripto-vivípara (Singh, 1998). En ambos tipos de germinación, el origen de la semilla es sexual, lo que las diferen- cia de la viviparidad cuyo origen es asexual (Beetle, 1980), también llamada viviparidad vegetativa (Batygina, 2005). Las plántulas vivíparas verdaderas pueden traspasar el pericarpio y liberarse, mientras que las cripto-vivíparas son incapaces de hacerlo, por lo que permanecen encriptadas hasta la dehiscencia del fruto o hasta que la pared del fruto es perforada por los consumidores. Elmqvist y Cox (1996) 
refieren que la cripto-viviparidad es una sub-categoría de la viviparidad verdadera. Antes de que ocurra la dispersión, las semillas maduras de tendencia vivípara o cripto-vivípara se mantienen activas fisiológicamente dentro del fruto (Farnsworth, 2000), lo que conlleva al crecimiento continuo del embrión y la germinación. Aun cuando el fruto no haya alcanzado la madurez o la dehiscencia y abscisión de la planta madre, en el proceso, el embrión aprovecha la humedad del fruto para continuar el desarrollo (Cota-Sánchez et al., 2007).

Los ejemplos más conocidos de viviparidad verdadera son los mangles, como Bruguiera gymnorrhiza (L.) Lamk., Rhizophora mangle L. (Tomlinson, 1986), R. stylosa Griff, Ceriops australis (C.T. White) Ballment,T.J. Sm \& Stoddart (Coupland et al., 2006), Aegiceras corniculatum (L.) Blanco (Ye et al., 2005), entre otras. En los ecosistemas de manglar se han identificado también especies cripto-vivíparas como Avicennia alba Blanco, A. marina (Forsk.) Vierh., A. officinalis L. (Raju et al., 2012) y A. germinans (L.) Stearn (Finney, 2011). De hábitats terrestres se ha mencionado viviparidad en varias familias botánicas como Arecaceae, Amaranthaceae, Cactaceae, Fabaceae, Liliaceae, Myrcinaceae, Pedialaceae y Poaceae (Cota-Sánchez et al., 2011; Antonucci et al., 2011; Prasad y Gangopadhyay, 2013).

Cerca de 60 especies de cactáceas producen plántulas cripto-vivíparas, una o dos por fruto, como en Lophocereus schottii (Engelm.) Britton \& Rose, y hasta más de doscientas plántulas por fruto, como en Epiphyllum phyllanthus (L.) Haw. (Cota-Sánchez y Abreu, 2007). Sin embargo, OrtegaBaes et al. (2010) no encontraron viviparidad en cactáceas del noroeste de Argentina, aun en aquellas que tenían semillas sin latencia, de donde concluyeron que el fenómeno debe ser muy raro y entraña una respuesta fisiológica más que adaptativa de las plantas. Sus puntos de vista difieren de Cota-Sánchez et al. (2011), quienes ubican a las cactáceas en cuarto lugar entre las familias de angiospermas con mayor proporción de especies vivíparas y sugieren evaluar la reproducción vivípara como adaptación a medios cambiantes y estrés fisiológico.

La diferenciación de las plantas vivíparas y pseudo-vivíparas en obligadas y facultativas (Batygina, 2005) implica diferente grado de especialización del sistema reproductivo. De acuerdo con esta autora, la viviparidad obligada se caracteriza por ser una adaptación necesaria para que las semillas crezcan y se desarrollen en la planta madre, con el fin de asegurar la supervivencia de los propágulos una vez dispersos en sitios permanentemente extremos; en Rhizophora mangle por ejemplo, la reproducción vivípara es obligada y representa una adaptación a la salinidad e inundación constantes. En el caso de la viviparidad facultativa, existe la capacidad reservada de las semillas para responder ante cambios ambientales inesperados, los cuales varían aleatoriamente en el tiempo, por lo que en respuesta, algunas semillas permanecen sin germinar mientras que otra fracción inicia el desarrollo de los propágulos hasta lograr plántulas viables cuando todavía permanecen en la planta madre.

La maduración de las semillas de mangles vivíparos coincide con una reducción de la concentración de ácido abscísico (ABA) y proteínas (dehidrinas) que protegen de la desecación a las membranas celulares (Farnsworth y Farrant, 1998; Farnsworth, 2000). Con estos cambios se incrementa notablemente el transporte de agua al embrión y se reduce el estrés osmótico, que son requeridos para el crecimiento continuo y precocidad en el desarrollo de la plántula. En opinión de Hoecker et al. (1995), y para el caso del maíz, existen activadores y represores de proteínas, como el activador transcripcional para viviparidad (VP1), cuya acción es inducir, en genes específicos, la producción de ABA, promover la tolerancia a la desecación, reducir el crecimiento del embrión y propiciar la maduración tardía. Es interesante considerar que, entre estas dos posibilidades funcionales, podría recaer la explicación de la viviparidad obligatoria o facultativa.

Los embriones vivíparos o cripto-vivíparos suelen desarrollarse y quedar libres al madurar los frutos, y ese proceso de liberación se facilita si los frutos son dehiscentes, como en el caso de Stenocereus thurberi (Engelm.) Buxb. (Arreola-Nava y Terrazas, 2003) y Epiphyllum phyllanthus, una cactácea epífita (Cota-Sánchez y Abreu, 2007; Simão et al., 2010). En el caso de Ferocactus herrerae J. G. Ortega, los frutos tienen un poro que abre en la base, por donde escapan las semillas y probablemente también las plántulas germinadas (Aragón-Gastélum et al., 2013).

Para el manejo y conservación de las semillas hasta la germinación es importante conocer su respuesta al secado y al almacenamiento (Dickie y Pritchard, 2002), pero la información es escasa. De acuerdo con estos autores, las semillas sensibles a la desecación pueden reducir su contenido de humedad hasta en $25 \%$ sin daño al embrión y, generalmente, no forman reservorios de semillas; tal es el caso de plantas acuáticas, del cacao (Toruan-Mathius et al., 2000) y varias especies de encino (Bonner y Vozzo, 1987). La respuesta fisiológica de estas especies es muy variable y se desconocen las condiciones óptimas para el almacenamiento, en general se recomienda almacenarlas a baja temperatura: 3 a $5^{\circ} \mathrm{C}$ en el caso de semillas de especies templadas y 7 a $17^{\circ} \mathrm{C}$ para las de especies tropicales (Magnitskiy y Plaza, 2007).

Algunos mangles vivíparos pueden perder de 30 a $60 \%$ de viabilidad de sus semillas a las $72 \mathrm{~h}$ de su recolección (Farnsworth y Farrant, 1998), por lo que debe trabajarse cuidadosa y rápidamente con este tipo de material. Las semillas de Avicennia alba con al menos $60 \%$ de humedad pueden ser recolectadas del árbol con todo y fruto para evitar la germinación precoz (Schmidt et al., 2004). La conservación del material durante el almacenamiento requiere reducir la humedad con secado hasta un 45\%, colocación en arena, 17 ${ }^{\circ} \mathrm{C}$ de temperatura y, después de cuatro meses, se observaría que el porcentaje de germinación ha declinado hasta el 
$75 \%$; aunque bajo esas condiciones es posible que algunas semillas germinen, proceso que puede limitarse si se baja la temperatura hasta cerca de $10{ }^{\circ} \mathrm{C}$, ya que menor temperatura sería letal para los embriones.

En cactáceas vivíparas faltan antecedentes sobre tolerancia a desecación y almacenamiento. Solamente se sabe que las semillas de Epiphyllum phyllanthus, altamente sensibles a la luz, pueden conservar su capacidad de germinación después de 12 meses de almacenamiento cuando son cosechadas antes de la maduración del fruto, secadas a $25^{\circ} \mathrm{C}$ y conservadas en frascos de vidrio a $10^{\circ} \mathrm{C}$ (Simão et al., 2010).

Stenocereus thurberi, la "pitaya dulce" que se trata en esta investigación, es una cactácea arborescente que se distribuye en el noroeste de México, en ambientes cálidos y semi-cálidos, secos a sub-húmedos con un período de estiaje; particularmente se localiza en el centro y sur de la península de Baja California, centro de Sinaloa, Sonora y algunas localidades de Chihuahua y Arizona en los Estados Unidos. $S$. thurberi forma parte de diferentes asociaciones vegetales como matorrales xerófitos y bosques espinosos de la zona costera del Pacífico y, en ocasiones se puede encontrar en el bosque tropical caducifolio, desde el nivel del mar hasta los $350 \mathrm{~m}$ de altitud (Arreola-Nava y Terrazas, 2003). Se considera que esta cactácea es vivípara facultativa porque sus poblaciones en el norte de Sinaloa tienen 88 a $95 \%$ de las plantas y 10 a $20 \%$ de los frutos afectados por viviparidad, usualmente con menos del $1 \%$ de las semillas germinadas en su interior (datos inéditos).

Los eventos de viviparidad facultativa que se han detectado en Stenocereus thurberi (Engelm.) Buxb. no están limitados a la zona de dunas costeras con influencia salina, también se presentan en suelos muy variados y vegetación típica de medios secos, como matorrales, bosques espinosos y selva baja caducifolia, donde impacta más la sequía y las altas temperaturas.

El objetivo de esta investigación fue comparar en Stenocereus thurberi, la capacidad germinativa de semillas remanentes en frutos con viviparidad vs. semillas procedentes de plantas sin evidencias de viviparidad, tanto de reciente cosecha en campo como después del almacenamiento bajo condiciones de laboratorio. Para ello se consideró importante saber: (1) si las semillas remanentes en frutos que contenían semillas vivíparas mostrarían diferencias en la respuesta germinativa, (2) si estas semillas mantendrían su capacidad germinativa después del almacenamiento y, (3) si esa capacidad germinativa post-almacenamiento de las semillas de frutos vivíparos, era estadísticamente comparable con la germinación de semillas de progenitores no vivíparos.

La hipótesis asume que las semillas de Stenocereus thurberi vivíparas facultativas son heteromórficas y confieren ventajas en la germinación y establecimiento de la progenie. Se esperaría encontrar un desarrollo diferencial en las semillas remanentes a causa de la precocidad, que las semillas procedentes de frutos con viviparidad perdieran capacidad germinativa al ser almacenadas por intolerancia al secado, y que en caso de haber germinación después del almacenamiento, esta fuera semejante en porcentaje, a la de semillas de plantas no vivíparas.

\section{Materiales y métodos}

Colecta de frutos y obtención de semillas. Los frutos fueron colectados en una población ubicada entre el matorral espinoso del pie de monte de la Sierra de Camayeca $\left(25^{\circ}\right.$ $59.5^{\prime} \mathrm{N}, 109^{\circ} 07^{\prime} \mathrm{O}$ ), cerca del Ejido Tosalibampo, en el norte de Sinaloa, México. El clima de esta localidad es seco desértico (BW), con una precipitación media anual de 419 $\mathrm{mm}$, temperatura media anual de $25.4^{\circ} \mathrm{C}$, máximas diarias superiores a $40^{\circ} \mathrm{C}$ en verano y mínimas de $2{ }^{\circ} \mathrm{C}$ en invierno (García, 1988). La reproducción de Stenocereus thurberi es unimodal de verano, la floración inicia en abril, alcanza el máximo en junio durante el período seco y concluye en agosto; la fructificación inicia en junio y termina en septiembre antes de las heladas.

Para determinar la condición de viviparidad se recolectaron y revisaron todos los frutos producidos entre junio y septiembre de 2010, de una muestra de 26 plantas. Más de mil frutos fueron inspeccionados con ayuda de un microscopio estereoscópico. Los hallazgos de semillas germinadas permitieron clasificar a las plantas en dos categorías reproductivas: vivíparas y no vivíparas. Se extrajo la pulpa de los frutos; las semillas se lavaron con agua corriente sobre una malla de $0.5 \mathrm{~mm}$ para separarlas del mucílago; después se dejaron secar y se almacenaron en bolsas de papel estraza a temperatura de $25^{\circ} \mathrm{C}$, hasta que fueron requeridas para los experimentos de germinación. Igual que Cota-Sánchez et al. (2007), se nombraron vivíparas a las plantas y frutos que tuvieron una o más semillas germinadas y no vivíparas a las que no mostraron evidencia de esto en todo el periodo de fructificación.

Las semillas seleccionadas para los experimentos proceden de seis plantas y fueron agrupadas en dos clases: a) las que se obtuvieron de plantas (3) cuyos frutos ninguno mostró evidencia de viviparidad en el periodo de maduración, clasificadas aquí en el grupo NV; b) las que se obtuvieron de plantas (3) con frutos afectados por germinación precoz, que fueron clasificadas en el grupo VV.

Experimentos de germinación. La germinación se evaluó en experimentos independientes, para diferentes fechas y condiciones de viviparidad, con las semillas NV como testigo experimental. En primer lugar, se comparó material colectado el 13 de septiembre, donde el grupo VV procedía de frutos homogéneos por tener igual nivel de viviparidad (2 semillas germinadas / fruto). En un experimento se usaron las semillas a las dos semanas de cosechadas y en otro a los 6.5 meses de almacenadas en seco. En segundo lugar, se diseñaron dos experimentos para comparar material colectado el 19 de julio y el 18 de agosto, donde el grupo vivíparo 
A
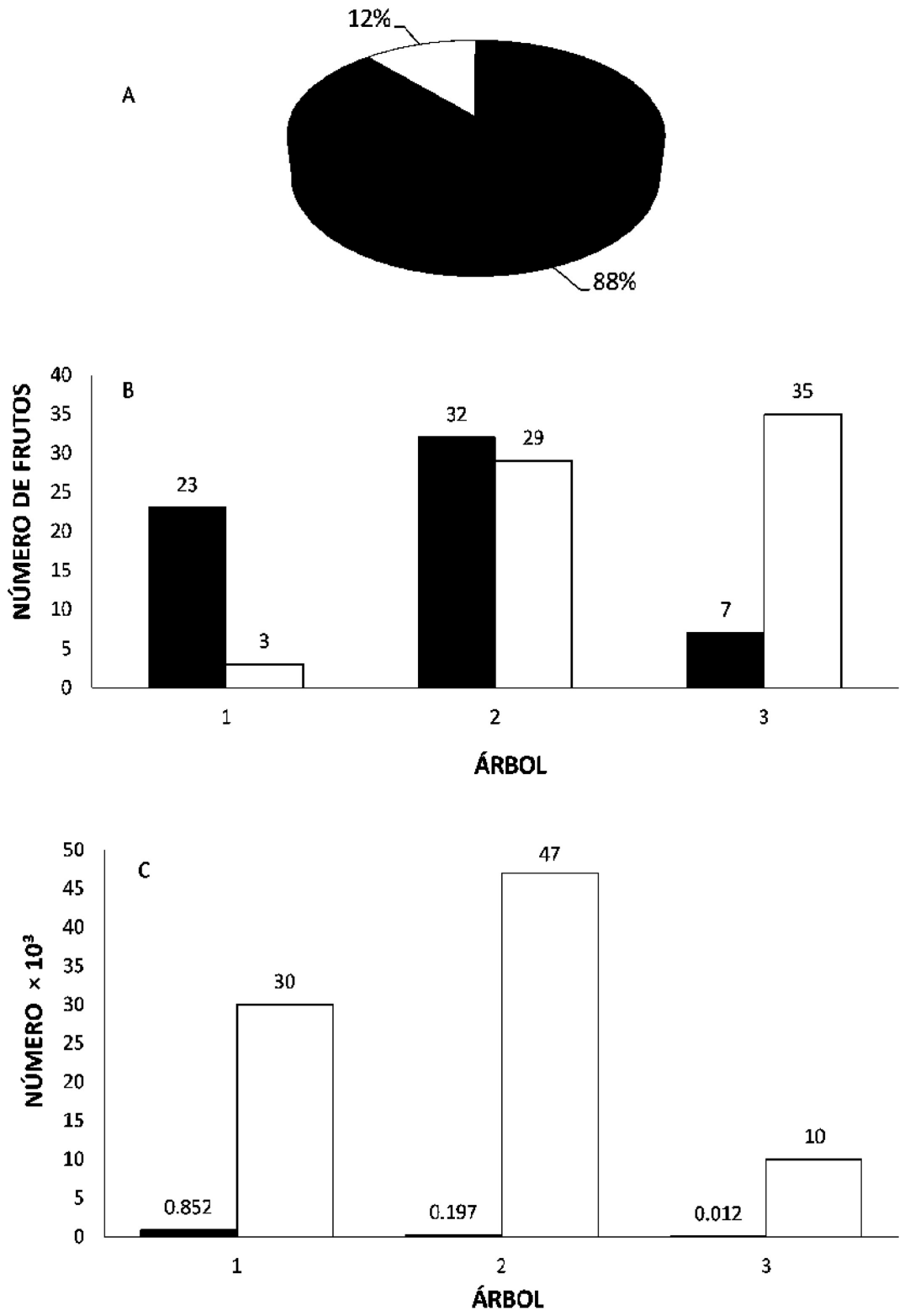

Figura 1. A) Plantas: $n=26, \mathbf{\square}=23$ con viviparidad; 934 frutos, 40.6 frutos por planta; $\square=3$ sin viviparidad; 98 frutos, 32.7 frutos por planta. B) Frutos de plantas vivíparas: $n=129, \boldsymbol{\square}=$ con semillas germinadas (VV), $\square=$ sin semillas germinadas, remanentes (NV). C)

Número de semillas en frutos con viviparidad $(\mathrm{n}=88,061)$ : $\mathbf{\square}=$ germinadas vivíparas, $\square=$ no germinadas remanentes (VV).

procedía de frutos heterogéneos por tener diferente nivel de viviparidad ( 2 a 352 semillas germinadas / fruto).

El procedimiento en todos los casos consistió en conformar unidades experimentales construidas con cajas plásticas transparentes de $24 \times 16 \times 14 \mathrm{~cm}$, que contenían $500 \mathrm{~g}$ de suelo tamizado con malla de $1 \mathrm{~mm}$, de la localidad de origen de las semillas. Por cada caja se colocaron 50 semillas de cada grupo (5 hileras de 10 semillas cada una). Las unidades experimentales se distribuyeron en diez bloques experimentales al azar, de acuerdo con las recomendaciones de la ISTA (2003), para evaluar la respuesta al almacenamiento. Las condiciones experimentales se registraron a intervalos de una hora con un data logger HOBO 8K TEMP/RH/LIGHT (Forestry Suppliers Inc., Jackson, Mississippi, E.U.A.). Se administraron fotoperiodos diarios de 15 horas luz con lámparas fluorescentes de $22 \mathrm{~W}\left(\sim 45.0 \mu \mathrm{mol} \cdot \mathrm{m}^{2} \cdot \mathrm{s}^{-1}\right)$, humedad a saturación $(\sim 30 \%)$ y temperatura constante de $25^{\circ} \mathrm{C}$, durante los 21 días del experimento.

Las semillas germinadas (con radícula o plúmula visible de $1 \mathrm{~mm}$ ), se registraron cada ocho horas los primeros tres días, y después cada 24 h durante 21 días. Las variables a evaluar correspondieron a tres momentos de la curva de germinación de las semillas: (1) el tiempo de inicio de la germinación $\left(\mathrm{T}_{\mathrm{i}}\right),(2)$ el tiempo medio de germinación o tiempo 
transcurrido entre la siembra y el 50\% de semillas germinadas con relación al total acumulado al final $\left(\mathrm{T}_{50}\right)$ y (3) el porcentaje acumulado de germinación o potencial germinativo (PG) a los 21 días. Tanto el $\mathrm{T}_{\mathrm{i}}$ como el $\mathrm{T}_{50}$ son indicadores de la velocidad de germinación.

Análisis estadísticos. Los datos del experimento con semillas homogéneas no se ajustaron a la distribución normal (prueba de Shapiro \& Wilk), por lo tanto se analizaron con un ANOVA no paramétrico de Kruskal-Wallis; las medianas de los tratamientos se compararon con la prueba estadística de WILCOXON para dos muestras, usando una aproximación a la normal estándar (Stokes et al., 2000). En el experimento con semillas heterogéneas, los tiempos de germinación $\mathrm{T}_{\mathrm{i}}, \mathrm{T}_{50}$ y PG, mostraron distribución normal y varianzas homogéneas (prueba $F$ de cociente máximo), por lo que se analizaron por medio de un modelo lineal (ANOVA de dos vías), y las medias de tratamientos se compararon con una $t$ de Student al 95\%. Los análisis se hicieron con los procedimientos UNIVARIATE, TTEST, GLM y NPAR1WAY, del programa estadístico SAS (SAS Institute Inc., 2004).

\section{Resultados}

De las 26 plantas muestreadas, $23 \mathrm{VV}$ y tres NV (Figura $1 \mathrm{~A})$, se obtuvo que por fruto se producen $1,472 \pm 71$ semillas (media \pm d.e.); la mayoría de los frutos $(\mathrm{n}=934$ de 1,032), presentó algunas semillas germinadas. Tres plantas fueron no vivíparas y produjeron de 20 a 44 frutos; en total se obtuvieron 98 frutos y más de dos millones de semillas. En las plantas identificadas como vivíparas (VV) se encontró una importante variación en la cantidad de frutos (Figura 1B) y semillas con viviparidad (Figura 1C).

La germinación de Stenocereus thurberi en ambos experimentos mostró diferencias significativas entre los dos tratamientos de viviparidad, tanto en semillas recién cosechadas como en las que fueron almacenadas por 6.5 meses (Figura 2). Las semillas con tendencia vivípara identificadas como VV germinaron un día antes, con mayor velocidad (dos días para lograr el 85\% de germinación vs. ocho días el otro caso, NV), y porcentaje germinativo mayor (97\%) que las semillas NV (92\%), procedentes de frutos que en ningún caso mostraron evidencias de viviparidad. Las semillas frescas VV iniciaron la germinación $31 \mathrm{~h}$ después de la siembra (1.25 días después de la siembra -dds-) y alcanzaron el 50\% de PG a las $48 \mathrm{~h}$. Las semillas testigo NV iniciaron a las 50 h (2.08 dds) y alcanzaron 50\% de PG a las 95 h (3.95 dds; Cuadro 1), lo que significa una diferencia de 19 horas en el $\mathrm{T}_{\mathrm{i}}$ y 24 horas en el $\mathrm{T}_{50}$, con respecto a las semillas VV. Las diferencias fueron significativas al $5 \%(Z>1.96)$ para las tres variables señaladas. Después de almacenarse por seis meses, la capacidad germinativa de las semillas VV disminuyó, mostrando $\mathrm{T}_{\mathrm{i}}$ de $43 \mathrm{~h}$ (1.8 dds), $\mathrm{T}_{50}$ de $62 \mathrm{~h}$ ( $2.6 \mathrm{dds}$ ) y PG de $95.5 \%$. Sin embargo, el testigo experimental NV mostró una disminución más rápida del vigor, al aumentar el
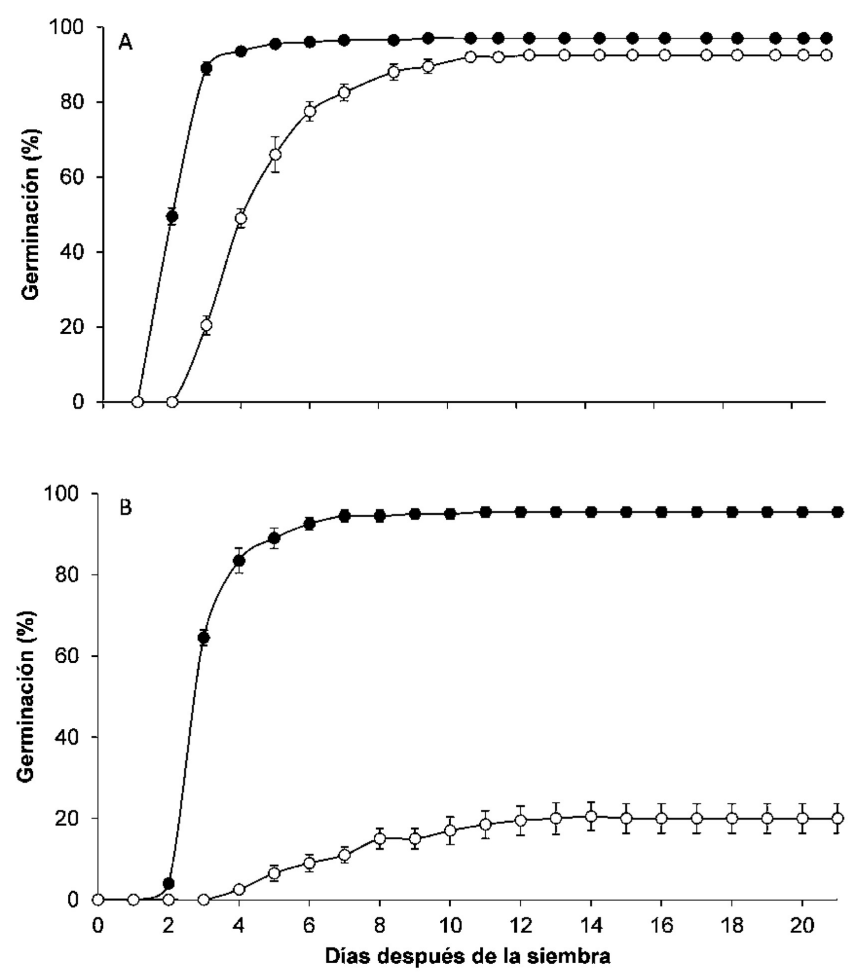

Figura 2. Germinación de semillas de Stenocereus thurberi (promedio \pm error estándar). A) Inmediatamente después de la cosecha y, B) después de seis meses de almacenamiento. manentes de frutos con viviparidad (VV); $O=$ semillas sin antecedente de viviparidad (NV). Las respuestas germinativas en $T_{i}, T_{50}$ y PG difieren al 0.05 con $\mathrm{Z}>11.96 \mid$ prueba de Wilcoxon, de acuerdo con el Cuadro 1.

$\mathrm{T}_{\mathrm{i}}$ a $89 \mathrm{~h}$ (3.7 dds), el $\mathrm{T}_{50}$ a $150 \mathrm{~h}$ (6.2 dds) al mismo tiempo que disminuyó el PG a $20 \%$.

Al comparar semillas heterogéneas del 19 de julio y del 18 de agosto en el segundo experimento, se observó amplia variación en la respuesta de germinación (Cuadro 2); debido a ello, no se encontraron diferencias en los tiempos de inicio de la germinación (3.1 vs $2.6 \mathrm{~d}, P=0.087$, prueba de $t$ ), $\operatorname{los} \mathrm{T}_{50}$ fueron más cortos en las semillas VV (4.9 vs 6.8, $P=0.017)$ y ambos tiempos se mostraron independientes de la incidencia de viviparidad $(P>0.05$, prueba de $F)$. Por el contrario, los porcentajes de germinación finales fueron mayores en semillas que no presentaron viviparidad (NV, $59.2 \%$ ), en comparación con las semillas que eventualmente pudiesen mostrar germinación precoz $(\mathrm{VV}, 43.2 \%, \mathrm{P}=$ 0.047), por lo que se relacionaron negativamente con el nivel de viviparidad $(P=0.027$, prueba de $F)$.

\section{Discusión}

Los individuos de Stenocereus thurberi del norte de Sinaloa presentan frutos con 500 semillas menos que la cantidad registrada por Parker (1987) en Arizona (1,969 semillas); el 
Cuadro 1. Respuesta germinativa (h, horas para la germinación), de semillas de reciente cosecha (2010) y de seis meses de almacenamiento (2011), colectadas de plantas de Stenocereus thurberi sin eventos de viviparidad en sus frutos (NV) y de plantas cuyos frutos tuvieron viviparidad $(\mathrm{VV}) . \mathrm{T}_{\mathrm{i}}=$ tiempo de inicio de la germinación; $\mathrm{T}_{50}=$ tiempo medio de germinación; PG = porcentaje final de germinación. Las respuestas difieren con $Z>|1.96|$, una aproximación a la distribución normal estándar de la Suma de Rangos de Wilcoxon; los valores de significancia corresponden a una prueba de dos colas.

\begin{tabular}{|c|c|c|c|c|c|}
\hline \multirow[t]{2}{*}{ Variable } & & \multicolumn{2}{|c|}{ Media \pm 1 error estándar } & \multirow{2}{*}{$\begin{array}{c}\text { Estadístico } \\
\text { Z }\end{array}$} & \multirow[t]{2}{*}{$\operatorname{Pr}>\mid Z$} \\
\hline & & NV & VV & & \\
\hline \multirow{3}{*}{2010} & $\mathrm{~T}_{\mathrm{i}}(\mathrm{h})$ & $50.8 \pm 0.42$ & $31.0 \pm 1.00$ & 2.23 & 0.0256 \\
\hline & $\mathrm{T}_{50}(\mathrm{~h})$ & $95.3 \pm 3.17$ & $48.1 \pm 1.09$ & 2.17 & 0.0304 \\
\hline & PG $(\%)$ & $92.5 \pm 0.96$ & $97.0 \pm 1.00$ & -1.97 & 0.0485 \\
\hline \multirow{3}{*}{2011} & $\mathrm{~T}_{\mathrm{i}}(\mathrm{h})$ & $89.0 \pm 3.79$ & $43.3 \pm 1.53$ & 2.20 & 0.0275 \\
\hline & $\mathrm{T}_{50}(\mathrm{~h})$ & $149.7 \pm 10.49$ & $62.1 \pm 0.91$ & 2.16 & 0.0304 \\
\hline & PG (\%) & $20.0 \pm 3.65$ & $95.5 \pm 1.26$ & -2.18 & 0.0294 \\
\hline
\end{tabular}

menor número de semillas puede deberse a que la población es marginal al área de distribución de la especie. Por otro lado, la criptoviviparidad de $S$. thurberi es una característica incipiente, a juzgar por los números: en plantas no vivíparas se producen cuatro veces más frutos por individuo que en plantas vivíparas, y estas muestran viviparidad en sólo el $1.22 \%$ del total de las semillas producidas, lo que significa que más del $95 \%$ de las semillas sigue el proceso de germinación propio para la especie, sin adelanto del proceso germinativo; en consecuencia, es importante destacar que las plantas vivíparas producen menos frutos pero tienden a crear una nueva expectativa biológica (o respuesta ecofisiológica), al anticipar el proceso germinativo y mantener el desarrollo continuo desde antes de la dispersión del fruto, en al menos, una pequeña fracción de semillas. Este hecho como estrategia de germinación, pudiese ser un factor importante para asegurar la supervivencia de la planta y la regeneración de las poblaciones en ambientes impredecibles (Keiffer y Ungar, 1997) y con ocasionales pulsos de humedad, como sucede en el Desierto Sonorense donde habita S. thurberi.

Con respecto al patrón de germinación de las semillas, se encontró que las semillas frescas remanentes de frutos

Cuadro 2. Respuesta germinativa (d, días) y potencial germinativo (\%) de las semillas de Stenocereus thurberi almacenadas por seis meses, en los tratamientos sin evidencia de viviparidad (NV, testigo) y semilla remanente de frutos con viviparidad (VV). LC= Límite de Confianza

\begin{tabular}{lcccc}
\hline Variable & $\begin{array}{c}\text { Origen de } \\
\text { la semilla }\end{array}$ & Media & LC95\% & $\begin{array}{c}\text { Mínimo - } \\
\text { Máximo }\end{array}$ \\
\hline Ti (d) & $\mathrm{NV}$ & 3.1 & $2.5-3.8$ & $2.33-5.00$ \\
& $\mathrm{VV}$ & 2.6 & $2.0-3.2$ & $1.33-3.67$ \\
& $\mathrm{NV}$ & 6.8 & $5.4-8.2$ & $4.29-9.40$ \\
$\mathrm{~T}_{50}(\mathrm{~d})$ & $\mathrm{VV}$ & 4.9 & $3.8-6.1$ & $2.84-8.08$ \\
& $\mathrm{NV}$ & 59.2 & $46.8-71.6$ & $32-92$ \\
$\mathrm{PG}(\%)$ & $\mathrm{VV}$ & 43.2 & $21.8-64.6$ & $4-98$ \\
& & & &
\end{tabular}

vivíparos (VV) germinaron en mayor porcentaje y velocidad que las semillas de progenitores vivíparos que tuvieron frutos no vivíparos (NV), aunque al final, el potencial germinativo se diferenció solo en 5\%. En contraste, la relación fue diferente y opuesta cuando se incorpora variación materna en fechas de maduración e incidencia de viviparidad. La tendencia que se encontró en las semillas remanentes corresponde con la precocidad y, por lo tanto, como se esperaba, habría un desarrollo diferencial entre semillas, reflejado en diferencias de capacidad germinativa. Además, se han observado diferencias morfológicas en lo que respecta al tamaño, lustre y redondez de la testa (Reyes Olivas, observación personal), pero hay una amplia variación que puede ser causada por otros factores no necesariamente relacionados con la viviparidad. La colecta anticipada al completo desarrollo del embrión puede influir en el tamaño de las semillas y por consecuencia, en la capacidad para germinar, como lo mencionaron Ayala-Cordero et al. (2004) para Stenocereus beneckii (Ehrenb.) Buxb.; también puede influir la condición materna y el ambiente (Gutterman, 2000).

De acuerdo con varios autores (McDonough, 1964; Nolasco et al., 1997; Sánchez-Soto et al., 2010), en condiciones de campo, la luz y temperatura menor de 15 o mayor de $40{ }^{\circ} \mathrm{C}$ afectan al potencial germinativo, haciéndolo variar entre 65 y $97 \%$; experimentalmente se ha mostrado que la germinación de Stenocereus thurberi, entre $\operatorname{los} 30-38^{\circ} \mathrm{C}$ promedia 88.5\% (Sánchez-Soto et al., 2005). En esta investigación se demostró que las semillas de plantas NV y VV de $S$. thurbe$r i$ logran altos porcentajes (92 y 97\%) de germinación a los $25^{\circ} \mathrm{C}$ que es la temperatura óptima para muchas especies de cactáceas (Nobel, 1988; Rojas-Aréchiga y Vázquez-Yanes, 2000; Castillo-Martínez et al., 2006). En otras cactáceas, el potencial germinativo puede lograrse con $27^{\circ} \mathrm{C}$, como es el caso de Echinopsis leucantha (Gillies ex Salm-Dyck) Walp., especie endémica de Mendoza, Argentina (Méndez y Pérez, 2008), o mediante escarificación de las semillas a $50{ }^{\circ} \mathrm{C}$, como en Mammillaria sphacelata Mart. de Puebla México (Navarro et al., 2008). 
Resaltamos tres aspectos importantes de las semillas remanentes de frutos con viviparidad (VV) en Stenocereus thurberi: (1) alta tolerancia a la desecación después de aislar a la semilla de la humedad del fruto, demostrada con un alto porcentaje de germinación aún después de seis meses de almacenamiento; (2) menor germinación asociada con alta incidencia de viviparidad; y (3) mayor velocidad para alcanzar el $50 \%$ de la germinación, dos días antes que las semillas sin antecedente de viviparidad.

Las cualidades señaladas contrastan con las semillas vivíparas de otras especies, que son característicamente intolerantes a la desecación y tienen alta capacidad germinativa. Por ejemplo, Inga saltensis Burkart (Fabaceae), una especie sudamericana, tiene $90 \%$ de germinación dentro del fruto y 95\% en semillas remanentes almacenadas por catorce días en el laboratorio (López-Spahr et al., 2011). Otra leguminosa sudamericana, Inga vera Willd. subesp. affinis (DC.) T.D.Penn., germina $95 \%$ dentro del fruto y pierde su viabilidad después de un mes, cuando la humedad de las semillas decae a 35\% (Faria, 2006). En mangles no vivíparos las semillas secas al aire son $100 \%$ viables después de $72 \mathrm{~h}$, mientras que en mangles vivíparos de Bruguiera la pérdida de viabilidad es del 60\%, en Aegialitis 50\% y en Aegiceres de $30 \%$. En la cactácea Echinocactus platyacanthus Link \& Otto, las semillas remanentes de frutos con viviparidad germinan entre 42 y $72 \%$, y las semillas que proceden de frutos no vivíparos germinan entre 14 y 51\% (Aragón-Gastélum, 2011); como puede advertirse, la superioridad de las semillas vivíparas no es consistente.

En cuanto a la velocidad de germinación, las semillas vivíparas de Stenocereus thurberi tardan dos días menos que las no vivíparas para alcanzar el 50\% de la germinación potencial; por lo tanto, la germinación es más rápida en las semillas que proceden de frutos donde hubo hallazgos de viviparidad; esta característica facilitaría la probabilidad de establecimiento en medios con precipitación intermitente o escasa (Dubrovsky, 1996, 1998; Yang et al., 2003). Las semillas de $S$. thurberi, igual que las de otras cactáceas, maduran y son liberadas desde principios del verano, antes de iniciar la estación lluviosa (Pimienta-Barrios y Nobel, 1998; Flores y Briones, 2001). En este contexto, la progenie vivípara y las semillas hermanas no germinadas dentro de los frutos tendrían mayor tiempo en la estación húmeda para convertirse en plántulas, con mayores reservas y vigor, cualidades que asegurarían la supervivencia durante la estación seca (Baskin y Baskin, 1998; Pritchard et al., 2004).

Se desconoce en Stenocereus thurberi si las semillas remanentes en frutos vivíparos tienen ventaja para la germinación bajo estrés hídrico, como se ha demostrado en otra cactácea vivípara del Altiplano Mexicano, Echinocactus platyacanthus. Las semillas vivíparas de esta especie, sembradas en un medio con potencial hídrico de $-0.4 \mathrm{MPa}$ tienen una tasa de germinación cuatro veces mayor que las semillas no vivíparas (47\% vs. 12\%; Aragón-Gastélum, 2011). La alta capacidad de germinación de las semillas de $S$. thurberi, después de 6.5 meses de almacenamiento, implica que si las semillas provienen de progenitores con semillas vivíparas, es probable que toleren la desecación. De esta forma, las semillas de $S$. thurberi manifiestan los dos atributos que usualmente son excluyentes en taxones recalcitrantes y vivíparos obligados, como son la germinación inmediata después de la dispersión y la alta tolerancia del embrión a la desecación de los tejidos. Un grupo de 28 especies (20 de Amaranthaceae), caracterizadas por tener germinación muy rápida (en menos de $24 \mathrm{~h}$ ), tienen semejanza con estos dos atributos y otras características de las semillas de Cactaceae (Parsons, 2012). Más recientemente, Liu et al. (2013) han reconocido que las cactáceas tienen características en común con las especies cripto-vivíparas; entre esas especies, cinco de ellas tienen semillas bifuncionales, latentes y vivíparas; la mayoría carecen de endospermo, tienen testas delgadas y se distribuyen en ambientes estresantes (árido, salino o inundable), donde pueden aprovechar rápidamente condiciones temporalmente favorables para la germinación.

Sarcobatus vermiculatus (Hook.) Torr. (Sarcobataceae, orden Caryophyllales), destaca por ser evidentemente vivípara, germina sobre la planta madre pero no se había mencionado como tal, ya que muchas semillas (24\%) tienen el ápice de la radícula expuesta y el crecimiento es continuo hasta formar una plántula en una hora (Eddleman, 1979); el resto de las semillas (76\%) requieren de uno a dos meses de post-maduración para germinar. Es probable que el bajo porcentaje de germinación en las semillas de Stenocereus thurberi, con antecedente de viviparidad menor al $50 \%$, esté relacionado con latencia morfo-fisiológica, en razón de que el embrión parece subdesarrollado o quizá esté fisiológicamente latente, de acuerdo con la clasificación explícita de Baskin y Baskin (2004) y la diagramada por Silveira (2013); sin embargo, lo anterior se deberá confirmar mediante estudios morfo-anatómicos, enzimáticos y hormonales involucrados en el desarrollo funcional de los embriones; de momento, se está de acuerdo en que hay un tiempo diferencial entre la germinación de las semillas de ambos grupos, que en términos de competencia y establecimiento facilita la permanencia sobre el sitio, con la ventaja de reducir la carga competitiva, y persistir aún, bajo las condiciones térmicas e hídricas severas que prevalecen al sur del Desierto Sonorense.

Las semillas del grupo no vivíparo germinaron bien al sembrarlas inmediatamente a la cosecha, en comparación con la respuesta posterior a los 6.5 meses de almacenamiento $(20 \%)$. Probablemente, la desecación de los tejidos o la temperatura de almacenamiento afectaron la funcionalidad temporal o definitiva de los tejidos embrionarios, causando latencia secundaria ante los cambios térmicos y de humedad, lo que implicaría que aún después de liberarlas de la condición de almacenamiento, no pudiesen germinar a pesar de ofrecerles condiciones favorables. Por esta respuesta, se 
acepta que hay germinación post-almacenaje al comparar las semillas de ambos tipos (NV y VV), con diferencias estadísticas en la capacidad germinativa. La respuesta de germinación post-almacenamiento registrada, no está generalizada en el grupo NV de Stenocereus thurberi, ya que otros autores (además de que no han referido viviparidad), han registrado $66 \%$ de germinación en semillas de uno a dos meses de cosecha (Yang et al., 2003), y 85 a 93\% en semillas de cinco meses (Sánchez-Soto et al., 2010). Para Epiphyllum phyllanthus, Simão et al. (2010) reconocen que aún después de 12 meses de almacenamiento, las semillas (sin precocidad) originadas de progenitores vivíparos, siguen siendo germinables.

Las semillas de Stenocereus thurberi ameritan mayor investigación porque tienen amplia variación en sus características fisiológicas, posiblemente asociadas con otros factores, independientemente de la condición vivípara / no vivípara; por ejemplo, que usualmente son fotoblásticas positivas (Sánchez-Soto et al., 2010). Se considera necesario precisar las relaciones entre las temperaturas de maduración y el patrón germinativo de las semillas, en especial de las especies que tienen frutos con semillas vivíparas y no vivíparas. Las respuestas de la germinación a la temperatura ayudarían a comprender el proceso de maduración y, sobre todo, a esclarecer el desarrollo de semillas en frutos que contienen semillas precoces y semillas no vivíparas; ya que al menos en las cactáceas arborescentes, la distribución de las ramas, que son las que en consecuencia sostienen a los frutos, varía con la altura y orientación de la planta madre y con la distribución de los frutos sobre las ramas (Roach y Wulff, 1987; Gutterman, 2000). Esta situación se ha verificado en Carnegiea gigantea (Drezner, 2003) y en Neobuxbamia tetetzo (López-Gómez et al., 2012). En consecuencia, la topología de las ramas pudiese establecer diferencias en la carga calórica recibida por los frutos y, como supone Cota-Sánchez (2008), probablemente algún proceso de termorregulación debe favorecer a la progenie vivípara; no podemos anticipar esta posible relación para el caso de las especies globosas como Coryphantha y Mammillaria, que también presentan semillas vivíparas, dado que en ese caso, la exposición de los frutos sigue otra topología. Independientemente de que se desconozca el origen de esta variación en cantidad y tiempo de germinación por efectos ambientales, parece claro que diferentes respuestas de germinación en tiempos y condiciones inesperadas, podrían representar la posibilidad alternativa para enfrentar la heterogeneidad ambiental que ocurre en espacio y tiempo (Wei et al., 2007).

Aún cuando estas diferencias parecen interesantes para la repoblación natural de Stenocereus thurberi, todavía se carece de suficientes datos sobre la variación del comportamiento germinativo entre frutos, plantas y poblaciones, lo cual ayudaría a precisar mejor el valor biológico de esta característica. Stenocereus thurberi, la cactácea estudiada bajo la comparación de semillas de frutos evidentemente sin eventos de viviparidad (NV) y frutos con semillas vivíparas (VV), mostró que hay diferencias en la velocidad de germinación y el vigor, pero no importantes en cuanto al potencial germinativo; es decir, la población de estas cactáceas de cualquier forma mantiene más del $90 \%$ del potencial germinativo, lo cual implica que la reproducción está asegurada fisiológica y ecológicamente. Esto da pie a continuar con las investigaciones y, lo que queda por probar, es que las semillas procedentes de progenitores con antecedente de viviparidad podrían eventualmente establecerse con más ventaja que las semillas no vivíparas y contribuir de manera importante en la demografía de la cactácea.

Tomando en cuenta el comentario de Ortega-Baes et al. (2010), acerca de que la viviparidad es más rara que común en la naturaleza, sería recomendable contribuir al conocimiento que coadyuve a aumentar la riqueza de los bancos de germoplasma con semillas almacenadas de germinación vivípara. Es pertinente continuar contribuyendo con la exploración de frutos recolectados en diferentes fechas y situaciones, para conocer la variación de las semillas, así como realizar más ensayos de germinación que permitan valorar diversos factores, ambientales y maternos, relacionados con el proceso de viviparidad y la respuesta germinativa de las semillas ante diversas condiciones de almacenamiento; ya que se reitera, la información en torno al almacenamiento y conservación de la capacidad germinativa de semillas vivíparas, escasamente se ha documentado.

\section{Agradecimientos}

El primer autor agradece el apoyo otorgado por el CONACYT (Reg. No. 247185) durante los estudios de maestría cursados en el Programa de Botánica del Colegio de Postgraduados, así como la colaboración en el trabajo de campo de O. Pérez-López y R. Nieblas-Ceceña. La investigación fue financiada por la Universidad Autónoma de Sinaloa, con fondos otorgados a uno de los autores (ARO) para el proyecto PROFAPI 2010-112. Se agradece a los revisores anónimos y al grupo editorial por la revisión y valiosa contribución aportada para la mejora de este documento.

\section{Literatura Citada}

Antonucci N.P., Abreu D.D. y Melo-de-Pinna G.F.A. 2011. Vivipary in Alternanthera littoralis var. maritime - first record for the Amaranthaceae. Botanica Marina 54:105-108.

Aragón-Gastélum J.L. 2011. Viviparidad en Echinocactus platyacanthus en el Altiplano Potosino y su posible beneficio para las etapas iniciales de desarrollo. Tesis de Maestría, Instituto Potosino de Investigación Científica y Tecnológica, San Luis Potosí, San Luis Potosí.79 pp.

Aragón-Gastélum J.L., Reyes-Olivas A., Sánchez-Soto B.H., Casillas-Álvarez P. y Flores J. 2013. Vivipary in Ferocactus herrerae (Cactaceae) in Northeastern Sinaloa, Mexico. Bradleya 31:44-52. 
Arreola-Nava H.J. y Terrazas T. 2003. Especies de Stenocereus con aréolas morenas: clave y descripciones. Acta Botanica Mexicana 64:1-18.

Ayala-Cordero G., Terrazas T., López-Mata L. y Trejo C. 2004. Variación en el tamaño y peso de las semilla y su relación con la germinación en una población de Stenocereus beneckei. Interciencia 29:692-697.

Baskin C.C. y Baskin J.M. 1998. Seeds. Ecology, Biogeography, and Evolution of Dormancy and Germination. Academic Press, San Diego.

Baskin J.M. y Baskin C.C. 2004. A classification system for seed dormancy. Seed Science Research 14:1-16.

Batygina T. 2005. Sexual and asexual processes in reproductive systems of flowering plants. Acta Biologica Cracoviensia Series Botanica 47:51-60.

Beetle A.A. 1980. Vivipary, proliferation, and phyllody in grasses. Journal of Range Management 33:256-261.

Bonner F.T. y Vozzo J.A. 1987. Seed biology and technology of Quercus. General Technical Report SO-66. U.S. Department of Agriculture, Forest Service, Southern Forest Experiment Station, Nueva Orleans.

Castillo-Martínez R., Brechú-Franco A.E., Cálix de Dios H. y Rojas-Aréchiga M. 2006. Germinación de Hylocereus undatus sometida a diferentes temperaturas y calidades de luz. Agrotecnología 5:31-37.

Cota-Sánchez J.H. 2008. Evolución de cactáceas en la región del Golfo de California. En: Flores C.L.M. Ed. Estudios de las Islas del Golfo de California, pp. 67-79, Universidad Autónoma de Sinaloa, Consejo Nacional de Ciencia y Tecnología, Gobierno del Estado de Sinaloa, Culiacán.

Cota-Sánchez J.H. y Abreu D.D. 2007. Vivipary and offspring survival in the epiphytic cactus Epiphyllum phyllanthus (Cactaceae). Journal of Experimental Botany 58:3865-3873.

Cota-Sánchez J.H., Reyes-Olivas Á. y Sánchez-Soto B. 2007. Vivipary in coastal cacti: a potential reproductive strategy in halophytic environments. American Journal of Botany 94:15771581.

Cota-Sánchez J.H., Reyes-Olivas Á. y Abreu D.D. 2011. Vivipary in the cactus family: A reply to Ortega-Baes' et al. evaluation of 25 species from northwestern Argentina. Journal of Arid Environments 75:878-880.

Coupland G.T., Paling E.I. y McGuinness K.A. 2006. Floral abortion and pollination in four species of tropical mangroves from northern Australia. Aquatic Botany 84:151-157.

Dickie J.B. y Pritchard H.W. 2002. Systematic and evolutionary aspects of desiccation tolerance in seeds. En: Black M. y Pritchard H.W. Eds. Desiccation and Survival in Plants: Drying Without Dying, pp. 239-259, CAB International, Nueva York.

Drezner T.D. 2006. Saguaro (Carnegiea gigantea) densities and reproduction over the northern Sonoran Desert. Physical Geography 27:505-518.

Dubrovsky J.G. 1996. Seed hydration memory in Sonoran Desert cacti and its ecological implication. American Journal of Botany 83:624-632.

Dubrovsky J.G. 1998. Discontinuous hydration as a facultative requirement for seed germination in two cactus species of the Sonoran Desert. Journal of the Torrey Botanical Society 125:33-39.

Eddleman L.E. 1979. Germination in black greasewood (Sarcobatus vermiculatus (Hook.) Torr.) Northwest Science 53:289294.
Elmqvist T. y Cox P.A. 1996. The evolution of viviparity in flowering plants. Oikos 77:3-9.

Faria J.M.R. 2006. Desiccation tolerance and sensitivity in Medicago trunculata and Inga vera seeds. Tesis Doctoral, Wageningen University, Wageningen. 133 pp.

Farnsworth E. 2000. The ecology and physiology of viviparous and recalcitrant seeds. Annual Review Ecology Systematics 31:107-138.

Farnsworth E.J.y Farrant M.J. 1998. Reductions in abscisic acid are linked with viviparous reproduction in mangroves. American Journal of Botany 85:760-769.

Finney W. 2011. Comparative growth and propagule viability of Louisiana-harvested black mangrove, Avicennia germinans. Tesis de Maestría en Ciencias Biológicas Marinas y Ambientales, Nicholls State University, Lafayette. 34 pp.

Flores J. y Briones O. 2001. Plant life-form and germination in a Mexican inter-tropical desert: effects of soil water potential and temperature. Journal of Arid Environments 47:485-497.

García E. 1988. Modificaciones al Sistema de Clasificación Climática de Köppen (para Adaptarlo a las Condiciones de la República Mexicana). $4^{\mathrm{a}}$ ed. Offset Larios, México, D.F.

Gutterman Y. 2000. Maternal effects on seeds during development. En: Fenner M. Ed. Seeds: The Ecology of Regeneration in Plant Communities. $2^{\mathrm{a}}$ ed., pp. 59-84, CAB International, Wallingford.

Hoecker U, Vasil I.K. y McCarty D.R. 1995. Integrated control of seed maturation and germination programs by activator and repressor functions of Viviparous-1 of maize. Genes and Development 9:2459-2469.

ISTA. International Seed Testing Association. 2003. International Rules for Seed Testing. Edición 2003. International Seed Testing Association, Zurich.

Keiffer C.H. y Ungar I.A. 1997. The effect of extended exposure to hypersaline conditions on the germination of five inland halophyte species. American Journal of Botany 84:104-111.

Liu K.; Baskin J.M., Baskin C.C. y Du G. 2013. Very fast-germinating seeds of desert species are cryptoviparous-like. Seed Science Research 23:163-167.

López-Gómez V., Zedillo-Avelleyra P., Anaya-Hong S. Y., GonzálezLozada E. y Cano-Santana Z. 2012. Efecto de la orientación de la ladera sobre la estructura poblacional y ecomorfología de Neobuxbaumia tetetzo (Cactaceae). Botanical Sciences 90:453-457.

López-Spahr D., Zapater-Cano M.A. y Ortega-Baes P. 2011. Viviparidad en Inga saltensis (Leguminosae). XXXIII Jornadas Argentinas de Botánica. Boletín de la Sociedad Argentina de Botánica 46:(Supl.): 105.

Magnitskiy S.V. y Plaza G.A. 2007. Fisiología de semillas recalcitrantes de árboles tropicales. Agronomía Colombiana 25:96103.

McDonough W.T. 1964. Germination responses of Carnegiea gigantea and Lemaireocereus thurberi. Ecology 45:155-159.

Méndez E. y Pérez G.S.B. 2008. Germinación de Echinopsis leucantha (Cactaceae). I- Efectos de temperatura y concentraciones de calcio. Revista de la Facultad de Ciencias Agrarias Universidad Nacional de Cuyo 40:91-96.

Navarro C.M.C., Cervantes O.G. y Lázaro C.J.O. 2008. Efecto de la escarificación de semillas en la germinación de dos especies de Mammillaria. Zonas Áridas 12:97-105.

Nobel P.S. 1988. Environmental Biology of Agaves and Cacti. Cambridge University Press, Nueva York. 
Nolasco H., Vega-Villasante F. y Díaz-Rondero A. 1997. Seed germination of Stenocereus thurberi (Cactaceae) under different solar irradiation levels. Journal of Arid Environments 36:123132.

Ortega-Baes P., Aparicio M. y Galíndez G. 2010. Vivipary in the cactus family: an evaluation of 25 species from northwestern Argentina. Journal of Arid Environments 74:1359-1361.

Parker K.C. 1987. Seed crop characteristics and minimum reproductive size of organ pipe cactus (Stenocereus thurberi) in Southern Arizona. Madroño 34:294-303.

Parsons R.F. 2012. Incidence and ecology of very fast germination. Seed Science Research 22:161-167.

Pimienta-Barrios E. y Nobel P.S. 1998. Vegetative, reproductive, and physiological adaptations to aridity of pitayo (Stenocereus queretaroensis, Cactaceae). Economic Botany 52:401-411.

Prasad R. y Gangopadhyay, G. 2013. 'Precocious' seed germination in Turkish and Chinese accessions of sesame (Sesamum indicum L.) in Indian conditions. American-Eurasian Journal of Agriculture and Environmental Sciences 13:398-401.

Pritchard H.W., Daws M.I., Fletcher B.J., Gaméné C.S., Msanga H.P. y Omondi W. 2004. Ecological correlates of seed desiccation tolerance in tropical African dryland trees. American Journal of Botany 91:863-870.

Raju A.J.S., Rao P.V.S., Kumar R. y Mohan S.R. 2012. Pollination biology of the crypto-viviparous Avicennia species (Avicenniaceae). Journal of Threatened Taxa 4:3377-3389.

Roach D.A. y Wulff R.D. 1987. Maternal effects in plants. Annual Review of Ecology and Systematics 18:209-235.

Rojas-Aréchiga M. y Vázquez-Yanes C. 2000. Cactus seed germination: a review. Journal of Arid Environments 44:85-104.

Sánchez-Soto B.H., García-Moya E., Terrazas T. y Reyes-Olivas Á. 2005. Efecto de la hidratación discontinua sobre la germinación de tres cactáceas del desierto costero de Topolobampo, Ahome, Sinaloa. Cactáceas y Suculentas Mexicanas 50:4-14.

Sánchez-Soto B.H., Reyes-Olivas Á., García-Moya E. y Terrazas T. 2010. Germinación de tres cactáceas que habitan la región costera del noroeste de México. Interciencia 35:299-305.
SAS Institute Inc. 2004. SAS/STAT ${ }^{\circledR} 9.1$ User's Guide. SAS Institute Inc.,Cary

Schmidt L.H., Le T.T.T. y Ho C.M.C. 2004. Avicennia alba Blume. Seed Leaflet 95:1-2

Silveira F.A.O. 2013. Sowing seeds for the future: the need for establishment protocols for the study of seed dormancy. Acta Botanica Brasilica 27:264-269.

Simão E., Nakamura A.T. y Takaki M. 2010. The germination of seeds of Epiphyllum phyllanthus (L.) Haw. (Cactaceae) is controlled by phytochrome and by nonphytochrome related process. Biota Neotropica 10:115-119.

Singh V.P. 1998. The new perspective in the halophyte. En: Agarwal S.K., Kaushik J.P., Koul K.K. y Jain A.K. Eds. Perspectives in Environment, pp. 189-204, S.B. Nangia \& A.P.H. Publishing Co., Nueva Delhi.

Stokes M.E., Davis C.S. y Koch G.G. 2000. Categorical Data Analysis Using the SAS System. 2a ed. SAS Institute Inc., Cary.

Tomlinson P.B. 1986. The Botany of Mangroves. Cambridge University Press, Nueva York.

Toruan-Mathius N., Rachmawati-Hasid N.H. y Hutabarat T. 2000. Physiological and biochemical changes in cocoa seed (Theobroma cacao L.) caused by desiccation. Menara Perkebunan 68:20-29.

Wei Y., Dong M. y Huang Z.Y. 2007. Seed polymorphism, dormancy and germination of Salsola affinis (Chenopodiaceae), a dominant desert annual inhabiting the Junggar Basin of Xinjiang, China. Australian Journal of Botany 55:464-470.

Yang X.Y., Pritchard H.W. y Nolasco H. 2003. Effects of temperature on seed germination in six species of Mexican Cactaceae. En: Smith R.D., Dickie J.B., Linington S.H., Pritchard H.W. y Probert R.J. Eds. Seed Conservation: Turning Science into Practice, pp. 575-588, The Royal Botanic Gardens, Kew, Londres.

Ye Y., Tam N.F.Y., Lu C.Y. y Wong Y.S. 2005. Effects of salinity on germination, seedling growth and physiology of three saltsecreting mangrove species. Aquatic Botany 83:193-205.

Recibido: 18 de octubre de 2013

Aceptado: 6 de febrero de 2014 